\title{
Advances in thermodynamics of solid surfaces
}

\author{
A.I.Rusanov \\ Chemistry Faculty, Ieningrad State University, Ieningrad 199034, USSR

\begin{abstract}
The thermodynamics of solid surfaces is the least developed part of the surface thermodynamics. The Gibbs adsorption equation referred only to fluid aystems, and an analogous equation for deformable solids was derived considerably later. Its general interpretation is complemented now by the concept of the tensorial anisotropic chemical potential corresponding to an anisotropic deformation state in a solid. Surface stresses produce the mechanical surface tension of which the direct measurement was a great problem up to now. The first method of measuring surface tension of solids has been proposed very recently and is based on the effect of deformation on solubility.
\end{abstract}

\section{INTRODUCTION}

The foundation of the thermodynemics of solid surfaces was elaborated by Gibbs (ref.1). His distinction of the work of formation of a unit surface, $\sigma$, from surface tension, $\gamma$, was an unexpected and important point of the theory determining the thought procession in the sequel. At the same time, the central statement of the theory of capillarity, the adsorption equation, was formulated by Gibbs only for fluid surfaces. Application to solids was considered by Bangham (ref.2). A generalization of the Gibbs adsorption equation for deformable solida was made considerably later: first by Eriksson for mechanically isotropic states (ref.3) and then by the author for a general anisotropic case, expressing the adsorption equation both through $\mathcal{E}$ and through $\gamma$ (ref.4). The step that took a century needed the understending of two things: a tensorial character of chemicel potentials in solids (essentially, it was pointed out by Gibbs, but seemed to be unthinkable since mass could not be a tensor) and that fact that the difference between $\sigma$ and $\gamma$ was due to the nonuniformity in chemical potentials at the surface of an even equilibrium solid. Both the points will be discussed in this communication.

In adition, of interest is a discussion (initiated in ref.5) of a practical role of $\dot{E}$ and $\gamma$ in various phenomena and of their measurement methods. The most known are theoretical estimations for comparisonbetween $\sigma$ and $\gamma$ (e.g., ref.6). As for experiment, the situation seems to be better with quantity $\sigma$ which participates in the Yung equation, and worse with quantity $\gamma$ for which, practically, there is no reliable data. Gokhshtein proposed a method for measuring variation in $\gamma$ ( $r e f .7$ ) and a method of a direct measurement of quantity o itself has been elaborated very recently (ref.8). Since this method has a thermodynamic foundation, it is also worthy to be described here.

\section{THE MODEL OF A COMPLETELY ELASTIC BODY AND ANISOTROPY OF CHEMICAL POTENTIAL}

The model of a completely elastic body is characterized by the presence of at least one immobile component forming an ideal (without defects) crystalline lattice. The lattice is capable of elastic deformation, but every given lattice site is occupied by a certein particle, so that diffusion of an immobile component is excluded. Mexwellian viscosity in such a model is infinite (if the elasticity modulus were also infinite, we would have the model of a completely rigid solid which is often used in the thermodynamics of wetting). Besides immobile components, the system may contein also mobile ones moving freely through the whole volume of the lattice, but their presence is not necessary.

The model of a completely elastic solid is a strictly equilibrium model to which the equetions of equilibrium thermodynamics ahould be applicable. However, a difficulty arises with the definition of chemical potential of an immobile component (if a body is uniform in composition, it is suffici- 
ent to consider only one immobile component (ref.1, p.194)). Chemical potential $\mu_{j}$ is defined usuelly as the derivative of energy $U$ with respect to the amolust of component $j, n_{j}$, at constant entropy $S$, constant volume $V$ of the system, and constant amounts $n_{i}$ of other components (mobile ones) if they are present:

$$
\mu_{j}=\left(\partial U / \partial n_{j}\right)_{S, V, n_{i}}
$$

In the case of en anisotropic body, the condition of constant volume should be understood as the condition of fixed boundaries of the system, but the difficulty is that the derivative expressed in (1) becomes dependent of $a$ finite state of deformation after a change in amount $n$. In other words, the energy of a solid in a given volume depends not only on mass, but aiso on the way of changing mass.

Recognizing this difficulty, Gibbs gave up introducing chemical potential for a solid and used only ordinary chemical potential $\mu_{j}^{\prime}$ of an immobile component in a dissolved state (when we speak "immobile" we mean the behavior of component $j$ in a solid, but component $j$ is capable of dissolution and behaves in a liquid phase as an ordinary component of solition) when a liquid solution is in equilibrium with a solid. Since pressure in a liquid is isotropic, such an equilibrium is possible only for three lattice planes in the solid perpendicular to the principal directions of the pressure tensor. Gibbs derived the equilibrium conditions for these directions (ref.1, p.194,217).

$$
\left(u_{v}-T s_{v}+p_{k}^{0}-\sum_{i} \mu_{i} \rho_{i}\right) / \rho_{j}=\mu_{j(k)}^{\prime}(k=1,2,3)
$$

where $\rho$ is molecular number density, $u_{v}$ energy density, $s_{v}$ entropy density, $p_{k}$ principal values of the pressure tensor, $\mu_{j}^{\prime}(k)$ equilibrium values of chemical potential of the immobile component in a liquid. The left-hand side of eq.(2) refers to a solid, the right-hand aide refers to a liquid phase, so eq. (2) is the condition of phase equilibrium. A more general condition of equilibrium for an arbitrary plane may be derived by changing $a$. liquid phase for a hypothetical solid phase where component $f$ behaves as a mobile one (ref.4):

$$
\left(u_{v}-T s_{v}+p_{\nu}-\sum_{i} \mu_{i} \rho_{i}\right) / \rho_{j}=\mu_{j(\nu)}^{\prime}
$$

where $p_{\nu}$ is pressure in direction $\vec{\nu}$, perpendicular to the plane under consideration, $\mu^{\prime}(\nu)$ corresponding value of chemical potential.

We return now to eq.(1) and answer the question: is it possible for a given solid to define a multitude of values of chemical potential related to different ways of introducing amount dnj into the system? There are the corresponding attempts in the literature (e.g., ref.8-11), Let us imagine that every differential of mass is characterized not only by its value, but also by its direction. If we add to $a$ solid the amount of its matter an $j(\nu)$ along the direction of unit vector $\vec{\nu}$, it means that the solid contracts in direction $\vec{\nu}$ and the empty volume formed is filled up with $\mathrm{dn}_{f(\nu)}$. Contraction in direction $\vec{\nu}$ may be represented as the simultaneous corresponding contractions in direction $x, y$ and $z$, so that three quantities $d n_{j(x)}, d n_{j(y)}$, and $d n_{j(z)}$ will completely determine $d n_{j}(\nu)$ Consequently, a directed change in emount $n$, may be generally characterized by tensor din. Respectively, chemical potential $\mu$ j $\nu$ for a given direction is defined as the derivative of energy with jrespect to amount $n_{j}$ along the same direction:

$$
\mu_{j}(\nu)=\left(\partial u / \partial n_{j}(\nu)\right)_{S, V, n_{i}}
$$

and the set of $\mu_{j(\nu)}$, makes the chemical potential tensor $\hat{\mu}_{j}$ of a solid. The fundamental equation for the energy of a uniform body may be written now in the form

$$
\mathrm{dU}=\mathrm{TdS}-(\hat{\mathrm{p}}: \mathrm{d} \hat{e}) \mathrm{V}+\hat{\mu}_{j}: \mathrm{d \hat {n } _ { j }}+\sum_{i} \mu_{i} \mathrm{~d} n_{i}
$$

where $T$ is temperature, $\hat{p}$ the pressure tensor, $\hat{e}$ the strain tensor. For $a$ given direction $\vec{\gamma}, \dot{q} \cdot(5)$ becomes

$$
\mathrm{dU}=\mathrm{TdS}-\mathrm{p}_{\nu} \mathrm{AdI}_{\nu}+\mu_{j(\nu)} \mathrm{dn_{j } ( \nu )}+\sum_{i} \mu_{i} d n_{i}
$$

where A is the constant along $\vec{\nu}$ cross-gection aree of the body, $I_{\nu}$ its dimension in direction $\overrightarrow{\mathcal{H}}$. After integretion at constant intengive vari- 
ables, we get from (6) the integral expression

$$
U=\mathbb{T S}-p_{\nu} V+\mu_{j}(\nu)^{n_{j}}+\sum_{i} \mu_{i} n_{i}
$$

or, in density terms,

$$
u_{v}=T s_{v}-p_{\nu}+\mu_{f(\nu)} \rho_{j}+\sum_{i} \mu_{i} \rho_{i}
$$

Comparing now (3) and (8), we obtain the phase equilibrium condition in the usual form

$$
\mu_{j(\nu)}=\mu_{j(\nu)}^{\prime}
$$

which confirms the meaning of quantity $\mu_{j(\nu)}$ as chemical potential.

Equation (8) may be written in the form

$$
\tilde{\omega}_{v}=-p_{\nu}+\mu_{j}(\nu) \rho_{j}
$$

where $\tilde{\omega}_{v}$ is the density of grend (with respect to mobile components) thermodynamic potential. $\widetilde{\Omega}$ (the free energy density in the absence of mobile components). Since the left-hand side of (10) does not depend on the direction, the combination of quantities on the right-hand side should be invariant with respect to direction.

\section{DIFFERENCE OF QUANTITIES $\sigma$ AND $\gamma$}

Up to now we discussed bulk properties of a solid, but the above concept of chemical potential is very important for understanding surface phenomena. Condition (9) means that chemical potentials of an immobile component in liquid and solid phases are equel only at the surfece of contact of the phases and does not mean that chemical potential is the same in different point of a solid. Just the reverse, chemical potential of a solid is unable to become uniform due to the absence of diffusion of an immobile component, so the nonuniformity of chemical potential may take place even in a truly equilibrium state of a completely elastic body. In particular, such nonuniformity exists always near the surface, which should be taken into account in calculations of excess quantities.

We consider this problem using eq.(10) as an example and passing to excess quantities for a flat dividing surface (perpendicular to the z-axis). The excess of the left-hend side of $(10)$ does not depend on direction and yields the known quantity $\sigma$ (excess free energy in the case of absence of mobile components):

$$
\sigma=\left(\tilde{\omega}_{v}-\tilde{w}_{v} \alpha, \beta\right) d z
$$

Superscript $\alpha$ refers to the bulk of a solid, $\beta$ refers to an adjacent phase, symbol $\alpha, \beta$ means $\alpha$ for $z<z_{0}$ and $\beta$ for $z>z_{0}$ where $z_{0}$ is the coordinate of the dividing surfece. The excess of $-p$, dependent of di-
rection $\vec{\nu}$ which now should be parallel to the surface (this follows from the fact that integration of $(6)$ is carried out along the direction of uniformity, 1.e. in any direction to the dividing surface if we deal with the surface layer), is the excess surface stress

$$
\gamma_{\nu}=-\int\left(p_{\nu}-p_{\nu}^{\alpha, \beta}\right) d z
$$

The excess of quantity $\mu_{j}(\nu) \rho_{j}$ may be represented as

$$
\begin{aligned}
& \int\left(\mu_{j(\nu)} \rho_{j}-\mu_{j(\nu)}^{\alpha, \beta} \rho_{j}^{\alpha, \beta}\right) d z=\mu_{j(\nu)}^{\alpha} \Gamma_{j}^{\alpha} \\
& +\mu_{j(\nu) \Gamma j}^{\beta}+\int\left(\mu_{j(\nu)}-\mu_{j(\nu)}^{\alpha, \beta}\right) \rho_{j} d z
\end{aligned}
$$

where $\Gamma^{\alpha, \beta}$ is the absolute adsorption of an immobile component on the side of phase $\alpha, \beta$. Thus, we obtain from (10) the relationship for ex-
cesa quantities

$\sigma=\gamma_{\nu}+\mu_{j(\nu)}^{\alpha} \Gamma_{j}^{\alpha}+\mu_{j(\nu)}^{\beta} \Gamma_{j}^{\beta}+\int\left(\mu_{j(\nu)}-\mu_{j(\nu)}^{\alpha, \beta}\right) \rho_{j} d z$ 
If chemical potentials $\mu_{j(\nu)}^{\alpha}$ and $\mu_{j(\nu)}^{\beta}$ in the adjacent phases $\alpha$ and $\beta$ are the same, or phase $\beta$ is a vacuum or a gas, eq. (14) takes the form

$$
G=\gamma_{\nu}+\mu_{j(\nu)}^{\alpha} \Gamma_{j}+\int\left(\mu_{j(\nu)}-\mu_{j(\nu)}^{\alpha} \rho_{j} d z\right.
$$

where $\Gamma g$ is the total adsorption of an immobile component. Eq.(15) is simplified if the dividing surface is chosed from condition $\Gamma_{j}=0$ :

$$
\sigma=\gamma_{\nu}+\int\left(\mu_{j(\nu)}-\mu_{j(\nu)}^{\alpha}\right) \rho_{j} d z
$$

In this case, quantity $\sigma$ is the same as in Gibbs' theory. Gibbs emphasized a difference between quentities $\sigma$ and $\gamma$ which exists only for solids, and eq.(16) gives a good explanation: absence of migration of an immobile component in a completely elastic solid (slowness of diffusion in a real solid) leads to nonuniformity in chemical potential and, as a consequence, to inequality of $\sigma$ and $\gamma$, which never takes place for an equilibrium liquid.

\section{MEASUREMENT OF $\gamma$}

It is known from the theory of elasticity that the free energy of an elastic body increases both during contraction and expansion if there is no stress in an initial state. In particular, free energy density must be the same on two sides of a bent elastic plate. In Gibbs' theory, the free energy density of a solid is uniquely related to chemical potential, so one may say that chemical potential on the opposite sides of the plate would be the same if there were no stress in an initial state. Actually such a stress exists: this is surface tension of a solid. Symmetry disappears in the case of a bent plate due to surface tension. Strain, free energy density, and chemical potentiel will be higher on the convex side then on the concave one if surface tension is positive, and lower on the convex side if surface tension is negative. The difference in chemical potential on two sides of a plate can be measured, for example, from the rate of dissolution of the sides or, directly, from the equilibrium concentrations of their saturated solutions.

It was established from experiments with plates of potassium chloride in water (ref.7) that the chemical potential of potassium chloride is higher on the concave side. This means that surface tension of potassium chloride is negative (the existence of a negative tension was predicted in some theoretical estimations ( $r e f, 6,9$ ). The value of the excess tangential pressure $\mathrm{p}_{\mathrm{T}}$ in the surface layer of a solid may be calculated from the formula (ref. 8 ).

$$
p_{T}=-\rho \Delta \mu / 2(1-\nu) e_{T}
$$

where $\rho$ is density of the solid, $\Delta \mu$ difference in the chemical potential of potassium chloride on the convex and concave plato sides, $\nu$ poisson's coefficient, $T_{T}$ tangential strain. One can pass from $p_{T}$ to surface tension $\gamma$ by means of relationship $\gamma=-p_{T} \tau$ where $\tau$ is the thickness of the surface layer being in equilibrium with the adjacent solution. The value obtained was $p_{T} \approx 4.105 \mathrm{~Pa}($ ref.7), and if we set $\tau \approx 3.10-10 \mathrm{~m}$ (the thickness of a monolayer of ions), we have $\gamma=-0,12 \mathrm{miN} / \mathrm{m}$ for the surface layer of potassium chloride in contact with the aqueous solution.

\section{REFERENCES}

1. The Scientific Pepers of J.W.Gibbs, Vol.1, p.315, Jongmans, New York $(1906,1931)$.

2. D.H.Bengham. Trans. Feraday Soc., 33, 805-811 (1937).

3. J.C.Eriksson. Surface Sci. 14, $221-246$ (1969).

4. A.I.Rusanov. J. Colloid Interfece Sci., 63, 330-345 (1978).

5. R. Shuttleworth. Proc. Phys. Soc., Iond., A 63, 444-457 (1950).

6. G.C.Benson and K.S.Yun in The Solid-Ges Interface (J.A.Flood, Ed.), Vol.1, p.203-271, Arnold, Jond.(1967).

7. A.Ya.Gokhshtein, Surfece Tension of Solids and Adsorption p.14, Nauka, Hoscow (1976).

8. G.V.Berenstein, A.ri. Dyachenko and A.I.Rusanov, Dokl. Acad. Na.uk SSSE $298,1402-1404$ (1988).

9. A.W.Adamson, Physical Chemistry of Surfaces, Ch.5, Wiley, New York (1976). 